\section{EXPERIÊNCIA DE 20 ANOS EM ADRENALECTOMIA VIDEOLAPAROSCÓPICA}

\section{TWENTY-YEAR EXPERIENCE IN LAPAROSCOPIC ADRENALECTOMY}

\author{
Renata Bruna Garcia dos Santos Gatelli', Alceu Migliavacca², \\ José Ricardo Guimarães ${ }^{2}$, Diego Mossmann², Leandro Totti Cavazzola ${ }^{3}$
}

\title{
RESUMO
}

Introdução: As operações na glândula adrenal são realizadas para determinados cânceres, todas as massas biologicamente ativas, metástases, massas com mais de 4-5 cm encontradas incidentalmente e hiperplasia adrenal primária.

Métodos: Estudo transversal e descritivo. Foram analisados os prontuários dos pacientes submetidos a adrenalectomia videolaparoscópica entre agosto de 1994 e novembro de 2014.

Resultados: Foram realizadas 146 adrenalectomias videolaparoscópicas. Em 134 casos, foi realizada com sucesso, mas em 12 casos $(8,2 \%)$, o procedimento foi convertido. Foram 97 pacientes do sexo feminino e 49 do sexo masculino, com idade variando de 9 a 81 anos (média de 46,7 anos). Foram removidas 56 adrenais direitas, 75 esquerdas e 15 bilaterais. $O$ tamanho médio das adrenais foi de $5,7 \mathrm{~cm}$, variando de 0,9 a $15 \mathrm{~cm}$. A mediana do tempo de internação hospitalar pós-operatória foi de 4,5 dias. A mediana do tempo de cirurgia foi de 144 minutos. Houve complicações em $22,5 \%$ dos casos (maiores - casos em que houve conversão para cirurgia aberta, necessidade de reinternação hospitalar e óbito- e menores), sendo 10,9\% complicações intraoperatórias e 11,6\% pós-operatórias. Apenas sete $(4,7 \%)$ pacientes foram considerados complicações maiores.

Conclusão: A cirurgia realizada em nosso serviço está de acordo com o descrito na literatura, com taxas aceitáveis de complicações, com motivos de conversão compatíveis e com as indicações totalmente aceitáveis e condizentes. A adrenalectomia videolaparoscópica é a cirurgia de escolha para patologias cirúrgicas da glândula adrenal, exceto em casos de carcinoma adrenal localmente invasivo com comprometimento de outras estruturas.

Palavras-chave: Glândulas suprarrenais; adrenalectomia; laparoscopia

\section{ABSTRACT}

Introduction: Adrenal gland surgery is performed for some types of cancer, all biologically active masses, metastases, masses larger than $4-5 \mathrm{~cm}$ found incidentally, and primary adrenal hyperplasia.

Methods: A cross-sectional, descriptive study. Medical records of patients who underwent laparoscopic adrenalectomy from August 1994 to November 2014 were analyzed.

Results: A total of 146 laparoscopic adrenalectomies were performed. In 134 cases, laparoscopic adrenalectomy was successfully performed, but in 12 cases (8.2\%), the procedure was converted. There were 97 female patients and 49 male patients. Fifty-six right adrenal glands and 75 left adrenal glands were removed, and 15 patients had both of them removed. The average size of adrenal glands was $5.7 \mathrm{~cm}$, ranging from 0.9 to $1.5 \mathrm{~cm}$. The median length of postoperative hospital stay was 4.5 days, ranging from 1 to 55 days. The median surgery time was 144 minutes. There were $22.5 \%$ of complications (major ones - cases that required conversion to open surgery, hospital readmission, and death - and minor ones), of which $10.9 \%$ were intraoperative and $11.6 \%$ were postoperative. Only seven $(4.7 \%)$ patients were classified as having major complications.

Clin Biomed Res. 2017;37(4):301-307

1 Serviço de Cirurgia Oncológica, Hospital de Clínicas de Porto Alegre. Porto Alegre, RS, Brasil.

2 Serviço de Cirurgia Geral, Hospital de Clínicas de Porto Alegre. Porto Alegre, RS, Brasil.

3 Departamento de Cirurgia, PósGraduação em Ciências Cirúrgicas, Universidade Federal do Rio grande do Sul (UFRGS). Porto Alegre, RS, Brasil.

Autor correspondente:

Renata Bruna Garcia dos Santos Gatelli renatabruna82@yahoo.com.br Hospital de Clínicas de Porto Alegre Rua Ramiro Barcelos, 2350 90035-903, Porto Alegre, RS, Brasil. 
Conclusion: The surgery performed in our department is consistent with the literature, showing acceptable rates of complications, compatible reasons for conversion, and completely acceptable and consistent indications. Laparoscopic adrenalectomy is the surgery of choice for diseases of the adrenal gland, except for locally invasive adrenal carcinoma compromising other structures.

Keywords: Adrenal glands; adrenalectomy; laparoscopy

As operações na glândula adrenal são realizadas para determinados cânceres, todas as massas biologicamente ativas, metástases, massas com mais de 4-5 cm encontradas incidentalmente e hiperplasia adrenal primária ${ }^{1}$. Com a realização de exames cada vez mais acurados, quase todos os tumores adrenais são identificados no pré-operatório, de modo que poucos casos exigem a exploração geral do abdome, o que possibilita a utilização da cirurgia minimamente invasiva ${ }^{2}$.

A escolha da abordagem cirúrgica depende de inúmeros fatores, entre eles a experiência/treinamento do cirurgião, a patologia e contraindicações para realização de videolaparoscopia. Com relação às características do tumor, a cirurgia aberta tem preferência quando a lesão for especialmente grande (> $12 \mathrm{~cm}$, dependendo da experiência do cirurgião) ou para o câncer adrenocortical localmente invasivo em que for necessário realizar linfadenectomia ou examinar órgãos adjacentes ${ }^{1,3}$.

A primeira adrenalectomia videolaparoscópica foi relatada em 1992 por Gagner et al. Desde então, vários estudos comparativos têm demonstrado as vantagens dessa técnica quando comparada com a cirurgia convencional por via aberta ${ }^{4-7}$.

A adrenalectomia laparoscópica pode ser realizada utilizando um acesso transabdominal ou retroperitoneal; porém, o primeiro é preferível, principalmente para tumores grandes ${ }^{1,8}$. A operação tradicional aberta deverá ser utilizada apenas quando a experiência laparoscópica não estiver disponível ou quando necessária em virtude da natureza e do tamanho do tumor ${ }^{1,9}$.

As vantagens da cirurgia laparoscópica são tão grandes que ela é fortemente preferida. Essa técnica permite melhor visualização da complexa anatomia da região, menos dor pós-operatória, menos tempo de internação e retorno mais rápido às atividades diárias. Há relatos na literatura de menos complicações operatórias, menor perda sanguínea, menor necessidade de transfusão de hemoderivados e melhor resultado cosmético ${ }^{10-12}$.

As complicações intraoperatórias mais descritas na literatura são: sangramento, lesões de outros órgãos e estruturas anatômicas, e pneumotórax. As pós-operatórias são: abscesso retroperitoneal, pneumonia e arritmias cardíacas ${ }^{5,12,13}$.

\section{MÉTODOS}

Este é um estudo transversal e descritivo. Foram analisados os prontuários dos pacientes, localizados pelos registros próprios da equipe e pelo sistema de informatização do hospital, submetidos a adrenalectomia videolaparoscópica entre agosto de 1994 e novembro de 2014. Um protocolo foi preenchido para cada paciente.

Neste estudo de casos, a amostra consistiu em pacientes submetidos a adrenalectomia videolaparoscópica no Hospital de Clínicas de Porto Alegre (HCPA), pela equipe de cirurgia oncológica, no período acima citado.

Foram incluídos os pacientes operados pelo serviço de cirurgia oncológica entre agosto de 1994 e novembro de 2014 e excluídos, os pacientes que não possuíam descrição cirúrgica completa e resultado anatomopatológico e aqueles operados por outros serviços.

Consideramos como critérios maiores de complicações cirúrgicas apenas os casos de necessidade de conversão para cirurgia aberta, necessidade de reinternação hospitalar e óbito.

Foi feita análise estatística utilizando o pacote estatístico IBM SPSS (Statistical Package for Social Sciences), versão 18.0 (Armonk, NY, IBM Corp).

As variáveis categóricas descritas foram: sexo, diagnóstico clínico e histopatológico, local do tumor, técnica cirúrgica, necessidade e motivo da conversão para cirurgia aberta, complicações intra e pós-operatórias. Foram avaliadas pelo teste qui-quadrado e teste exato de Fischer quando indicado.

As variáveis contínuas foram: idade, peso, altura, tamanho do tumor, permanência no hospital e tempo cirúrgico. Foram descritas por média, mediana e desvio padrão.

O projeto foi aprovado pela Plataforma Brasil e pelo Comitê de Ética em Pesquisa (CEP) do HCPA.

No acesso transperitoneal, técnica cirúrgica realizada neste trabalho (Figura 1), os pacientes foram posicionados em decúbito lateral. O pneumoperitôneo foi obtido através de colocação de trocarte de $10 \mathrm{~mm}$, através da técnica aberta, com pequena incisão transversa situada na linha hemiclavicular na altura da cicatriz umbilical e insuflado dióxido de carbono $\left(\mathrm{CO}_{2}\right)$ na pressão de $12-14 \mathrm{mmHg}$ e colocada a câmera. Três portais adicionais de 5 ou 


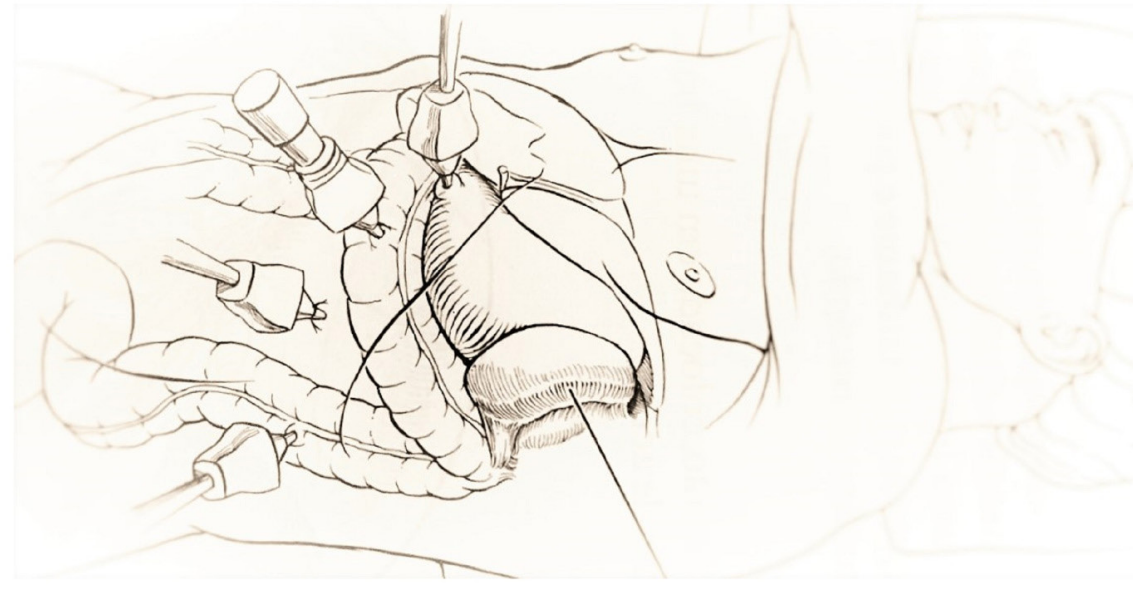

Figura 1: Acesso transperitoneal - posicionamento dos trocartes ${ }^{14}$.

$10 \mathrm{~mm}$ foram colocados: na linha hemiclavicular, aproximadamente dois dedos abaixo da margem costal, na linha axilar anterior, na altura da cicatriz umbilical, e na linha axilar posterior.

$\mathrm{Na}$ adrenalectomia direita, o peritônio foi incisado e rebatido inferiormente, e o fígado foi afastado para exposição adequada. A dissecção da glândula adrenal direita começou de forma inferior e lateral. A veia adrenal entrando na cava foi identificada e dissecada precocemente, e foi então duplamente ligada com clipes metálicos e seccionada. A dissecção prosseguiu superiormente no tecido adiposo entre a glândula adrenal e a face lateral da veia cava inferior. Os vasos encontrados foram cuidadosamente ligados. A dissecção continuou inferiormente até a face inferomedial da glândula, quando foi liberada da fáscia de Gerota. A glândula adrenal foi removida em um saco endoscópico através de um dos portais. Foi então realizada a revisão da hemostasia.

Para o acesso à adrenal esquerda, a flexura esplênica foi incisada e o cólon foi rebatido inferiormente. A dissecção do tecido ao redor da face posterior da cauda do pâncreas ajudou a definir a borda anterior da adrenal esquerda. A dissecção continuou de forma posteroinferior no tecido fibroadiposo entre a adrenal e o rim, e prosseguiu anteriormente com atenção para a localização da veia adrenal esquerda.

\section{RESULTADOS}

No período de agosto de 1994 a novembro de 2014, foram realizadas 146 adrenalectomias por videolaparoscopia no serviço de cirurgia oncológica do HCPA. Em 134 casos, o procedimento foi realizado com sucesso, mas em 12 casos foi convertido devido a dificuldade técnica (sete casos), sangramento (quatro casos, sendo que em um caso houve lesão da artéria renal) e pneumotórax (um caso). Nessas conversões, quatro tumores eram maiores do que $10 \mathrm{~cm}$, seis eram maiores do que $7 \mathrm{~cm}, 11$ eram maiores do que $5 \mathrm{~cm}$ e um apresentava $2,6 \mathrm{~cm}$.

Em nosso estudo, 80 pacientes $(54,7 \%)$ tinham adrenal maior do que $5 \mathrm{~cm}$ e, desses, 11 (13\%) tiveram as cirurgias convertidas para via aberta convencional, ou seja, a grande maioria dos casos $(86 \%)$ foi operada por via laparoscópica. Acima de $8 \mathrm{~cm}$, a taxa de conversão foi $40 \%$. A adrenal foi maior do que $12 \mathrm{~cm}$ em três casos, com taxa de conversão de $66 \%$ (dois casos) (Figura 2).

Foram 97 pacientes do sexo feminino e 49 do sexo masculino, com idade variando de 9 a 81 anos (média de 46,47 anos). Foram removidas 56 adrenais direitas, 75 esquerdas e 15 bilaterais. O tamanho médio das adrenais foi de $5,7 \mathrm{~cm}$, variando de 0,9 a $15 \mathrm{~cm}$.

O tempo médio de internação hospitalar pós-operatória foi de 7 dias, variando de 1 a 55 dias, com mediana de 4,5 dias. O tempo médio de cirurgia foi de 169,51 minutos, variando de 80 a 350 minutos, com mediana de 144 minutos (Tabela 1).

As doenças adrenais foram 74 adenomas, 28 feocromocitomas, 19 hiperplasias, sete carcinomas, quatro ganglioneuromas, três metástases, dois focos inflamatórios crônicos linfoplasmocitários, duas paracoccidioidomicoses, dois mielolipomas, dois nódulos acessórios microscópicos e de extrusão 


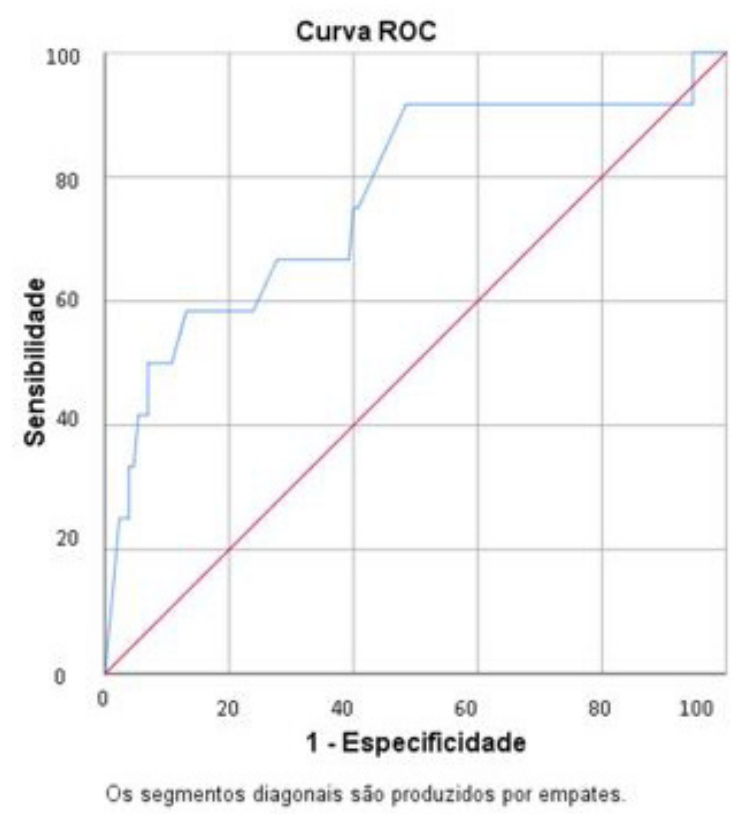

Figura 2: Curva ROC (receiver operating characteristic curve) para o tamanho da adrenal e a conversão para cirurgia aberta. cortical focal microscópica, um hemangioma cavernoso, um infarto hemorrágico e um pseudocisto adrenal.

Houve um óbito, que ocorreu no pós-operatório imediato e com atestado de óbito evidenciando choque hipovolêmico. Além disso, houve 16 pacientes com complicações pós-operatórias, sendo cinco com hematoma, três com infecção em ferida operatória, um com fístula pancreática, um com abscesso retroperitoneal, um com tromboembolismo pulmonar, um com trombose da veia renal, um com aumento da pressão intraocular, um com derrame pleural bilateral, um com isquemia cerebral occipitotemporal direita e um com lesão do plexo celíaco/dor neuropática. Três pacientes precisaram de reinternação hospitalar, um por abscesso retroperitoneal, um por celulite e um por insuficiência adrenal (Tabela 2).

Dos 12 casos em que houve conversão para cirurgia aberta, quatro foram diagnosticados como adenoma, quatro como carcinoma, um como feocromocitoma, um como hiperplasia, um como ganglioneuroma e um como mielolipoma.

Tabela 1: Adrenalectomia laparoscópica no HCPA - dados demográficos, adrenal acometida, tamanho da adrenal, dias da cirurgia até a alta e tempo de cirurgia.

\begin{tabular}{|c|c|c|c|c|c|}
\hline & $\mathbf{N}(\%)$ & Média & Mediana & Mínimo & Máximo \\
\hline Total & $146(100)$ & & & & \\
\hline Idade & & 46,47 & 47,00 & 9 & 81 \\
\hline Peso (kg) & & 72,06 & 71,30 & 40 & 131 \\
\hline Altura $(\mathrm{m})$ & & 1,6226 & 1,6100 & 1,45 & 1,90 \\
\hline IMC & & 27,44 & 28,00 & 17 & 49 \\
\hline Tamanho da adrenal $(\mathrm{cm})$ & & 0,9 & 15,0 & 5,75 & --- \\
\hline Dias da cirurgia até a alta & & 1 & 55 & 6,92 & 4,5 \\
\hline Tempo de cirurgia (min) & & 80 & 350 & 169,5 & 144 \\
\hline Homem & $49(33,5)$ & & & & \\
\hline Mulher & $97(66,4)$ & & & & \\
\hline Adrenal direita acometida & $56(38,3)$ & & & & \\
\hline Adrenal esquerda acometida & $75(51,3)$ & & & & \\
\hline Adrenal bilateral acometida & $15(10,1)$ & & & & \\
\hline
\end{tabular}

Tabela 2: Adrenalectomia laparoscópica no HCPA -complicações intraoperatórias.

\begin{tabular}{ccc}
\hline & Categoria & $\mathbf{N}(\%)$ \\
\hline Total & Não & $146(100)$ \\
Complicações intraoperatórias & Sangramento & $130(89,04)$ \\
& Hematoma hepático & $10(6,8)$ \\
& Lesão da artéria renal esquerda e baço & $1(0,68)$ \\
$1(0,68)$ & $1(0,68)$ & $1(0,68)$ \\
& Abertura do delgado & $1(0,68)$ \\
& Perfuração do diafragma & $1(0,68)$ \\
\hline
\end{tabular}


Tabela 3: Adrenalectomia laparoscópica no HCPA - causas de conversão para técnica aberta e valor dep.

\begin{tabular}{cccc}
\hline & Conversão para cirurgia aberta & $\%$ & Valor dep \\
\hline Total & 12 & 100 & \\
Tumores $<12 \mathrm{~cm}$ & 10 & 83,3 & 0,933 \\
Tumores $>12 \mathrm{~cm}$ & 2 & 16,6 & 0,001 \\
Carcinoma adrenal & 4 & 3,3 & 0,001 \\
IMC $>30$ & 4 & 3,3 & 0,324 \\
\hline
\end{tabular}

IMC = índice de massa corporal.

Com relação às causas de conversão para técnica aberta, foi estatisticamente significativa a conversão em tumores maiores que $12 \mathrm{~cm}$ e nos diagnosticados como carcinoma da glândula adrenal. Em tumores menores que $12 \mathrm{~cm}$ e nos pacientes com índice de massa corporal (IMC)> 30, não houve diferença estatisticamente significativa (Tabela 3).

Para estimar a curva de aprendizado, dividimos cronologicamente a amostra de adrenalectomias unilaterais em dois grupos de 64 pacientes e comparamos tempo de operação, diâmetro máximo da lesão e duração da internação. Não houve diminuição no tempo médio de operação após a obtenção de mais experiência. Observou-se uma tendência para a remoção de lesões maiores. Não houve diferença significativa em termos de internação hospitalar.

\section{DISCUSSÃO}

Desde que foi descrita pela primeira vez, em 1992, por Gagner et al. ${ }^{9}$, a adrenalectomia videolaparoscópica vem ganhando força e tornou-se a cirurgia padrão-ouro para ressecção da glândula adrenal. Inúmeros trabalhos foram publicados, porém não há consenso com relação às indicações cirúrgicas quanto ao diagnóstico e ao tamanho das lesões. Muitos autores defendem a sua realização para tumores benignos e pequenos, sem comprovação de que a técnica videolaparoscópica não seja factível quando evidenciado o diagnóstico de carcinoma adrenal.

A adrenalectomia é indicada para diversas doenças, incluindo as massas ativas, a hiperplasia da glândula, o feocromocitoma, o carcinoma, as metástases e o incidentaloma com mais de $4 \mathrm{~cm}$ de diâmetro.

A dificuldade técnica imposta em tumores com tamanho acima de $12 \mathrm{~cm}$ foi um dado observado em nosso trabalho, já que as conversões para cirurgia aberta se deram em $66,7 \%$ desses tumores, resultado estatisticamente significativo $(p=0,001)$ e de acordo com os dados da literatura. Dos casos operados em nosso serviço, três apresentavam lesões acima de $12 \mathrm{~cm}$, sendo que houve conversão para cirurgia aberta em dois deles. Já nos tumores menores do que $12 \mathrm{~cm}$ (97,9\% dos casos), não houve essa correlação estatisticamente significativa entre o tamanho e a conversão para cirurgia aberta $(p=0,933)$.

Dos 146 pacientes estudados em nosso trabalho, 12 precisaram ser convertidos para cirurgia aberta $(8,2 \%)$, sendo que em quatro casos a causa da conversão foi sangramento, um por pneumotórax e sete por dificuldade técnica. Nas conversões devido a sangramento, os tumores apresentavam mais de $6 \mathrm{~cm}$, o que nos leva a concluir que a dificuldade em dissecar a adrenal é maior nesses tumores. O cirurgião fica com um campo mais restrito e com uma dificuldade maior para identificar, dissecar e ligar os vasos envolvidos na adrenalectomia. Relatos na literatura evidenciam que lesões vasculares, especialmente da veia cava, compreendem quase $7 \%$ de todas as complicações da adrenalectomia laparoscópica e são a principal causa de conversão para cirurgia aberta ${ }^{14}$.

Rieder et al. ${ }^{8}$ tentou comprovar que a adrenalectomia direita poderia ser mais desafiadora devido à localização retrocaval da glândula e à anatomia da veia adrenal (mais curta quando comparada à veia adrenal esquerda), porém não conseguiu provar que a ressecção da glândula direita impõe ao cirurgião uma dificuldade maior que o lado contralateral. Nesse estudo, a adrenalectomia esquerda demandou um tempo cirúrgico maior devido à estreita proximidade com a cauda do pâncreas, à vascularização do baço e à natureza implacável do baço em si. Varkarakis et al. ${ }^{15}$ relataram uma taxa de $8 \%$ de lesão distal do pâncreas com a adrenalectomia laparoscópica. O lado esquerdo também requer a dissecção do hilo renal esquerdo para ganhar controle vascular da veia renal, o que potencialmente aumenta a complexidade do processo do lado esquerdo ${ }^{8}$. Em nossa casuística, não houve maior taxa de conversão nas adrenalectomias direitas, o que corrobora os dados descritos até agora.

Outro dado a ser considerado é a técnica operatória. Em nosso trabalho, todos os casos foram realizados pela técnica transperitoneal, que fornece um campo mais amplo quando comparada à técnica retroperitoneal. Há maior acesso aos vasos envolvidos na dissecção da glândula, motivo pelo qual optamos por essa técnica em nosso serviço. 
Com relação à conversão para a técnica aberta, $33,3 \%$ dos casos (quatro pacientes) ocorreram em pacientes com diagnóstico de carcinoma da glândula adrenal. Houve necessidade de conversão em $57 \%$ dos casos diagnosticados como câncer de adrenal, uma causa estatisticamente significativa $(p=0,001)$ quando comparada às taxas de conversão nas neoplasias benignas da glândula adrenal; desses, $50 \%$ apresentaram sangramento intraoperatório. Gagner et al. consideram ocarcinoma adrenal invasivo umacontraindicação absoluta para a abordagem laparoscópica devido à possível extensão e complexidade da operação requerida (ressecção em bloco do rim com a gordura perirrenal e baço, dissecção de linfonodos) $)^{9}$. Porém, em nossa opinião, o câncer de adrenal não impede a cirurgia por videolaparoscopia, pois a contraindicação está relacionada à dificuldade técnica do cirurgião e não à patologia em si. Tumores grandes são factíveis de ser ressecados por essa técnica, e trabalhos até agora publicados descrevem adrenalectomias laparoscópicas para tumores malignos primários da adrenal com margens negativas e sem implantes nos locais dos trocartes ${ }^{16,17}$. Não houve implante tumoral nos locais de inserção por trocartes em nenhum dos pacientes operados por carcinoma de adrenal em nosso serviço.Fossa et al.fizeram um estudo recente envolvendo 32 pacientes com carcinoma adrenal nos estágios I-III, dos quais 17 foram submetidos a adrenalectomia laparoscópica e 15 a adrenalectomia aberta $^{18}$. Os dois grupos foram semelhantes em metástases locais, peritoneais e distantes. Em uma das maiores séries (152 pacientes) com tumores $<10 \mathrm{~cm}$, Brix et al. observaram recidiva tumoral e carcinomatose peritoneal, respectivamente, em $77 \%$ e $3 \%$ dos pacientes após adrenalectomia laparoscópicaversus 69\% e 3\% dos pacientes apósadrenalectomia aberta; a diferença, no entanto, não foi estatisticamente significativa ${ }^{19}$.

Não existe consenso na literatura quanto à contraindicação à técnica laparoscópica em função do tamanho da adrenal, porém Gagner et al. ${ }^{9}$ evidenciaram que massas $>15 \mathrm{~cm}$ são mais bem abordadas por via aberta. Outros afirmam que, nos tumores maiores que $10 \mathrm{~cm}$, está contraindicada a adrenalectomia laparoscópica ${ }^{20}$. Em nosso trabalho, foi estatisticamente significativa a conversão em tumores a partir de $5 \mathrm{~cm}$ $(p=0,007)$, o que está de acordo com a evidência de que grandes lesões impõem dificuldade técnica ao cirurgião.

Em nosso estudo, apesar de utilizarmos a curva ROC (receiver operating characteristic curve), não conseguimos estipular um ponto de corte para o tamanho da adrenal associado à conversão para cirurgia aberta convencional. Verificamos que, dos 12 pacientes que tiveram suas cirurgias convertidas para via tradicional aberta, 11 possuíam adrenal maior do que $5 \mathrm{~cm}$, porém com uma taxa de conversão de apenas $13 \%$, já que 69 pacientes (86\%) foram operados por videolaparoscopia. Em pacientes com adrenal acima de $8 \mathrm{~cm}$, a taxa de conversão foi de $40 \%$ e, em casos acima de $12 \mathrm{~cm}$, essa taxa subiu para $66 \%$. Fica evidente a relação entre o tamanho da glândula e a taxa de conversão, mesmo sem a identificação de um ponto de corte.

O IMC do paciente não foi um empecilho para a realização da técnica fechada, já que 39 pacientes apresentaram IMC acima de 30 , mas apenas quatro $(10,25 \%)$ destes evoluíram para cirurgia aberta, sem significância estatística $(p=0,324)$. Petris et al. ${ }^{21}$ e Guazzoni et al. ${ }^{22}$ evidenciaram a superioridade da cirurgia laparoscópica quanto a perda sanguínea, complicações, mobilização rápida, melhor aspecto cosmético e recuperação mais rápida, em pacientes obesos, assim como nós, já que não encontramos diferenças entre pacientes com IMC $<30$ e $\geq 30$ quanto a tempo operatório, complicações, conversões e permanência hospitalar.

Tivemos $22,5 \%$ de complicações (maiores e menores), sendo $10,9 \%$ de complicações intraoperatórias e 11,6\%, pós-operatórias. Foram considerados na categoria de complicações maiores apenas sete $(4,7 \%)$ pacientes, já que apenas nesses casos houve necessidade de conversão para cirurgia aberta (cinco casos), necessidade de reinternação hospitalar (um caso) e óbito (um caso). Em uma meta-análise retrospectiva, Brunt et al. ${ }^{23}$ avaliaram as complicações de 1.633 adrenalectomias relatadas em 50 estudos. $O$ índice total de complicações foi de $25,2 \%$. Mancini et al. ${ }^{24}$ estudaram 172 pacientes e obtiveram $8,7 \%$ de complicações. Outros estudos, com séries de mais de 50 casos cada um, obtiveram de 0 a $28 \%$ de complicações, segundo diferentes critérios para definir complicações ${ }^{25-26}$.

Com relação à curva de aprendizado, não houve diminuição do tempo cirúrgico médio quando dividimos a amostra de adrenalectomias unilaterais em dois grupos; porém, ocorreu uma tendência para a remoção de lesões maiores, o que pode ter contribuído para que houvesse aumento no tempo cirúrgico.

Pela experiência da equipe cirúrgica, a contraindicação à técnica proposta está em lesões francamente aderidas a outras estruturas, em que não seria possível uma retirada em bloco da peça, considerada cirurgia padrão-ouro para a ressecção da grande maioria dos tumores.

A cirurgia realizada em nosso serviço está de acordo com o descrito na literatura, com taxas aceitáveis de complicações, com motivos de conversão compatíveis e com as indicações totalmente aceitáveis e condizentes. Assim, a adrenalectomia videolaparoscópica é a cirurgia de escolha para doenças cirúrgicas da glândula adrenal, exceto em casos de carcinoma adrenal localmente invasivo com comprometimento de outras estruturas. 


\section{REFERÊNCIAS}

1. Trahan MD. Suprarrenais - anterior, posterior (aberta e laparoscópica). In: Townsend CM, Evers BM. Atlas de técnicas cirúrgicas. São Paulo: Elsevier Ltda; 2011. p. 88-105.

2. Duh QY, Liu C, Tyrrell JB. Suprarrenais. In: Doherty GM. Cirurgia: diagnóstico e tratamento. Rio de Janeiro: Guanabara Koogan Ltda; 2010. p. 684-5.

3. Venkatasubramanian R, Wadhwa A, Sharma A, Khullar R, Soni V, Baijal $M$, et al. Laparoscopic adrenalectomy - a review of initial 24 consecutive patients. Indian J Surg. 2007;69(4):12935. PMid:23132964. http://dx.doi. org/10.1007/s12262-007-0003-7.

4. Hisano M, Vicentini FC, Srougi M. Retroperitoneoscopic adrenalectomy in pheochromocytoma. Clinics (Sao Paulo). 2012;67(S1 Suppl 1):1617. PMid:22584723. http://dx.doi. org/10.6061/clinics/2012(Sup01)27.

5. Dalvi AN, Thapar PM, Shah NS, Menon PS. Has experience changed the scenario in laparoscopic adrenalectomy? Indian J Surg. 2009;71(2):78-83. PMid:23133120. http://dx.doi.org/10.1007/s12262-0090021-8.

6. Poulin EC, Schlachta CM, Burpee SE, Pace KT, Mamazza J. Laparoscopic adrenalectomy: pathologic features determine outcome. Can J Surg. 2003;46(5):340-4. PMid:14577705.

7. Nunes LF, Mello ELR, Corrêa JHS. Análise crítica da adrenalectomia videolaparoscópica: experiência do INCA e revisão da literatura. Rev Bras Cancerol. 2003;49:215-20.

8. Rieder JM, Nisbet AA, Wuerstle MC, Tran VQ, Kwon EO, Chien GW. Differences in left and right laparoscopic adrenalectomy. JSLS. 2010;14(3):36973. PMid:21333190. http://dx.doi.org/10. 4293/108680810X12924466007520.

9. Gagner M, Pomp A, Heniford BT, Pharand D, Lacroix A. Laparoscopic adrenalectomy: lessons learned from 100 consecutive procedures. Ann Surg. 1997;226(3):238-47. PMid:9339930. http://dx.doi.org/10.1097/00000658199709000-00003.

10. Carter Y, Mazeh H, Sippel R, Chen $\mathrm{H}$. Safety and feasibility of laparoscopic resection for large ( $\geq 6$ $\mathrm{CM})$ Pheochromocytomas without suspected malignancy. Endocr Pract. 2012;18(5):720-6. PMid:22982788. http://dx.doi.org/10.4158/EP12014.OR

11. Humphrey R, Gray D, Pautler S, Davies W. Laparoscopic compared with open adrenalectomy for resection of pheochromocytoma: a review of 47 cases. Can J Surg. 2008;51(4):276-80. PMid:18815650

12. Edwin B, Kazaryan AM, Mala T, Pfeffer PF, Tønnessen TI, Fosse E. Laparoscopic and open surgery for pheochromocytoma. BMC Surg. 2001;1(1):2. PMid:11580870. http:// dx.doi.org/10.1186/1471-2482-1-2.

13. Ramachandran MS, Reid JA, Dolan SJ, Farling PA, Russell CFJ. Laparoscopic adrenalectomy versus open adrenalectomy: results from a retrospective comparative study. Ulster Med J. 2006;75(2):126-8. PMid:16755942.

14. Corcione F, Esposito C, Cuccurullo D, Settembre A, Fusco F, Bianco A, et al. Vena cava injury. A serious complication during laparoscopic right adrenalectomy. Surg Endosc. 2001;15(2):218. PMid:12200664

15. Varkarakis IM, Allaf ME, Bhayani SB, Inagaki T, Su LM, Kavoussi $L R$, et al. Pancreatic injuries during laparoscopic urologic surgery. Urology. 2004;64(6):1089-93. PMid:15596174. http://dx.doi.org/10.1016/j. urology.2004.06.032.

16. Kebebew E, Siperstein AE, Duh QY. Laparoscopic adrenalectomy: the optimal surgical approach. $J$ Laparoendosc Adv Surg Tech A. 2001;11(6):409-13. PMid:11814133. http://dx.doi. org/10.1089/10926420152761941.

17. Heniford BT, Arca MJ, Walsh RM, Gill IS. Laparoscopic adrenalectomy for cancer. Semin Surg Oncol. 1999;16(4):293-306. PMid:10332775. http://dx.doi.org/10.1002/(SICl)10982388(199906)16:4<293::AIDSSU4>3.0.CO;2-E.

18. Fosså A, Rosok BI, Kazaryan AM, Holte HJ, Brennhovd B, Westerheim O, et al. Laparoscopic versus open surgery in stage I-III adrenocortical carcinoma: a retrospective comparison of 32 patients. Acta Oncol. 2013;52(8):1771-7. PMid:23398621. http://dx.doi.org/10.310 9/0284186X.2013.765065.
19. Brix D, Allolio B, Fenske W, Agha $A$, Dralle $\mathrm{H}$, Jurowich $\mathrm{C}$, et al. Laparoscopic versus open adrenalectomy for adrenocortical carcinoma: surgical and oncologic oucome in 152 patients. Eur Urol. 2010;58(4):609-15. PMid:20580485. http://dx.doi.org/10.1016/j. eururo.2010.06.024.

20. Henry JF, Defechereux T, Gramatica L, Raffaelli M. Should laparoscopic approach be proposed for large and/ or potentially malignant adrenal tumors? Langenbecks Arch Surg. 1999;384(4):366-9. PMid:10473857. http://dx.doi.org/10.1007/ s004230050215.

21. Paun D, Petris R, Ganescu R, Paun S, Vartic M, Beuran M. Outcome of Laparoscopic Adrenalectomy in Obese Patients. Maedica (Buchar). 2015;10(3):231-6. PMid:28261359.

22. Guazzoni G, Cestari A, Montorsi F, Lanzi R, Nava L, Centemero $A$, et al. Eight-year experience with transperitoneal laparoscopic adrenal surgery. J Urol. 2001;166(3):8204. PMid:11490226. http://dx.doi. org/10.1016/S0022-5347(05)65844-2.

23. Brunt LM. The positive impact of laparoscopic adrenalectomy on complications of adrenal surgery. Surg Endosc. 2002:16(2):2527. PMid:11967673. http://dx.doi. org/10.1007/s00464-001-8302-8.

24. Mancini F, Mutter D, Peix JL, Chapuis Y, Henry JF, Proye C, et al. Expérience de la surrénalectomie en 1997. Á propos de 247 cas. Étude prospective multicentrique de l'Association francophone de chirurgie endocrinienne. Chirurgie. 1999;124(4):368-74. PMid:10546389. http://dx.doi. org/10.1016/S0001-4001(00)80008-2.

25. Thompson GB, Grant CS, vanHeerden JA, Schlinkert RT, Young WF JR, Farley $\mathrm{DR}$, et al. Laparoscopic versus open posterior adrenalectomy: a casecontrol study of 100 patients. Surgery. 1997;122(6):1132-6. PMid:9426429. http://dx.doi.org/10.1016/S00396060(97)90218-X.

26. Henry JF, Defechereux T, Raffaelli M, Lubrano D, Gramatica L. Complications of laparoscopic adrenalectomy: results of 169 consecutive procedures. World J Surg. 2000;24(11):1342-6. PMid:11038204. http://dx.doi. org/10.1007/s002680010222.

Recebido: Jun 06, 2017 Aceito: Nov 10, 2017 